\title{
EVIDÊNCIAS DE REGENERAÇÃO NATURAL EM ÁREA DEGRADADA DENOMINADA CASCALHEIRA-TRÊS
}

\author{
LAGOAS/MS
}

\author{
Maria José Neto ${ }^{1}$
}

Rodrigo Martins dos Santos ${ }^{2}$

\begin{abstract}
Rony Carlos Barcelos Blini ${ }^{3}$
RESUMO

A destruição de habitats por empreendimentos humanos, dentre os quais a formação dos reservatórios para usinas hidrelétricas, pode levar ao desaparecimento de espécimes biológicos de grande importância. Um exemplo é a área denominada Cascalheira utilizada para a remoção de cascalho durante a construção da Usina Hidrelétrica Eng. Souza Dias na década de 1960. As evidências de regeneração natural deste local permitem a correção de eventuais problemas quanto à criação de uma base de dados que permita avaliar e refinar as estratégias prescritas para a restauração de áreas degradadas. Com objetivo de evidenciar possíveis graus de regeneração natural, procedeu-se o levantamento florístico da referida área, localizada no município de Três Lagoas-MS. A pesquisa foi realizada em campanhas trimestrais durante o ano de 2013 e a metodologia utilizada foi a de caminhamento. Os espécimes coletados foram depositados no Herbário-CEUL. Os dados obtidos permitiram identificar 136 espécimes, 135 em nível de espécie e uma até nível de gênero, distribuídas em 107 gêneros e 50 famílias. Com relação às origens ou ocorrências, $72,79 \%$ ocorrem naturalmente no Brasil, e quanto ao habitat ocupado 68 espécies são terrestres, 52 de brejos e 16 tipicamente aquáticas. Os resultados permitem concluir que a regeneração natural vem ocorrendo no local.
\end{abstract}

PALAVRAS-CHAVE: regeneração natural, levantamento florístico, cascalheira

\section{EVIDENCE OF NATURAL REGENERATION IN DEGRADED AREA CALLED CASCALHEIRA (TRES LAGOAS / MS - BRAZIL)}

\author{
ABSTRACT \\ ${ }_{1}^{1}$ Prof. ${ }^{a}$ Dra em Agronomia - Universidade Federal do Mato Grosso do Sul. maria.neto@ufms.br \\ ${ }^{2}$ Graduando em C.Biológicas - Universidade Federal do Mato Grosso do Sul - rodrigoms13@hotmail.com \\ ${ }^{3}$ Mestrando em Geografia - Universidade Federal do Mato Grosso do Sul ronyblini@hotmail.com
}


Habitat destruction by human endeavors, among them the formation of reservoirs for hydroelectric plants, can lead to the disappearance of biological specimens of great importance. An example is the area called Cascalheira used for removing gravel during the construction of Hydroelectric Engo Souza Dias in 1960. Evidence of natural regeneration of this site allow correction of any problems regarding the creation of a database that evaluate and refine strategies prescribed for the restoration of degraded areas. In order to highlight possible degrees of natural regeneration, proceeded to the floristic survey of that area located in Tres Lagoas-MS. The survey was conducted on a quarterly campaigns during the year 2013 and the methodology used was the pathway. The specimens collected were deposited in the Herbarium-CEUL. It was possible to identify 136 specimens, 135 species-level and one to the genus level, distributed in 107 genera and 50 families. Regarding the origins or occurrences, $72.79 \%$ naturally occurring in Brazil, and in relation to habitat occupied 68 species are terrestrial, 52, and 16 typically waterfowl wetlands. The results show that natural regeneration is occurring on site.

KEYWORDS: natural regeneration, floristic study, cascalheira.

\section{LA EVIDENCIA DE LA REGENERACIÓN NATURAL EN ÁREAS DEGRADADAS LLAMADO CASCALHEIRA-(TRES LAGOAS / MS - BRASIL)}

\section{RESUMEN}

La destrucción del hábitat por actividades humanas, entre ellas la formación de embalses para centrales hidroeléctricas, puede conducir a la desaparición de las muestras biológicas de gran importancia. Un ejemplo es la zona denominada Cascalheira utiliza para quitar la grava durante la construcción de Hidroeléctrica Ing. Souza Dias en 1960. Evidencia de la regeneración natural de este sitio permite la corrección de cualquier problema con respecto a la creación de una base de datos que evaluar y perfeccionar las estrategias establecidas para la restauración de áreas degradadas. Con el fin de poner de relieve los posibles grados de la regeneración natural, se procedió a la encuesta de la flora de esa zona ubicada en Tres Lagoas-MS. La encuesta se realizó en una campañas trimestrales durante el año 2013 y la metodología utilizada fue la vía. Los especímenes recolectados fueron depositados en el Herbario-CEUL. Los datos obtenidos permitieron identificar 136 ejemplares, 135 a nivel de especie y uno a nivel de género, distribuidas en 107 géneros y 50 familias. En cuanto a los orígenes o las ocurrencias, 72,79\% ocurren de forma natural en el Brasil; sobre el hábitat ocupado, 68 especies son terrestres, 52 especies son del pantano y 16 son típicamente acuáticas. Los resultados muestran que la regeneración natural se está produciendo en el sitio

PALABRAS CLAVE: regeneración natural, el estudio florístico, cascalheira

\section{INTRODUÇÃO}

A destruição de habitats por empreendimentos humanos, dentre os quais a formação dos reservatórios para usinas hidrelétricas, tem propiciado o desaparecimento de muitas espécies vegetais e animais em níveis alarmantes 
(FERREIRA et al., 2009). Superfícies ocupadas por áreas de empréstimo, depósito de material rochoso e de "bota-foras" dos canteiros de obras de hidrelétricas, formam setores alterados, cuja recuperação torna-se um problema considerável. Essas áreas de um modo geral são exploradas com equipamentos de larga escala, que vão compactando o solo, permanecendo sem qualquer condição para que nele se processe a regeneração natural, conforme Ferreira et al. (2010).

Complementando, Fonseca et al. (2013) dizem que a regeneração natural poderá decorrer da interação de processos de restabelecimento do ecossistema; sendo assim, parte do ciclo de crescimento da floresta irá referir-se nas fases iniciais de estabelecimento e desenvolvimento.

De acordo com Faria et al. (2001), para que ocorra regeneração natural, em áreas totalmente degradadas ou em processo de degradação, são necessárias algumas condições como o cessar dos processos causadores da degradação, entre eles pastoreio e incêndios, a existência de fonte de propágulos (banco de sementes do solo, chuva de sementes), presença de dispersores, boas condições microclimáticas e edáficas, ausência de predadores e agentes antrópicos, para o estabelecimento e ocorrência do ciclo de vida completo das plântulas. Quanto aos processos de avaliação, enfatizou Rodrigues (1999) que a regeneração natural pode, entre outros, ser avaliada por meio de um levantamento florístico.

A sucessão vegetal permite a retomada da dinâmica sucessional formando uma paisagem harmônica e estável, propagando o recobrimento do solo. A tendência da sucessão é avançar expandindo e ocupando espaços vazios, atraindo animais dispersores de sementes, que utilizam a vegetação como ponto de pouso, de refúgio, de descanso e de alimentação e, com isso, garantindo a eficiência da regeneração natural no processo de sucessão, favorecendo a biodiversidade no local. (CAMPELLO et al, 1997; SILVA, 2005).

Conforme Carvalho (1992), o estudo da regeneração natural permite a realização de previsões sobre o comportamento e desenvolvimento futuro da floresta, pois fornece a relação e a quantidade de espécies que constituem o seu estoque e distribuição na área. Silveira e Maranho (2012) afirmaram que a regeneração natural depende da característica de cada espécie, em perfeita sintonia com as condições 
ambientais, sendo as propriedades do solo de grande importância para os ecossistemas terrestres.

Para Fonseca et al. (2013), monitorar essas comunidades que se formam em áreas recuperadas é de suma importância, devendo ser efetuada tanto para permitir a correção de eventuais problemas quanto para a criação de uma base de dados que permitam avaliar e refinar as estratégias prescritas para a restauração de áreas degradadas.

Um exemplo de área em recuperação natural é a Cascalheira, objeto de estudo deste trabalho, utilizada para a remoção de cascalho durante a construção da Usina Hidrelétrica Eng. Souza Dias na década de 1960. Devido à proximidade dos lençóis freáticos da região, os buracos escavados na época se encheram de água formando lagoas próximas aos remanescentes da vegetação original (SOUZA, 2007). Nesse contexto, o objetivo deste trabalho foi verificar evidências de regeneração natural por meio do levantamento florístico das lagoas e suas proximidades da referida área.

\section{OBJETIVOS GERAIS}

Verificar evidências de regeneração natural por meio do levantamento florístico das lagoas e suas proximidades, da referida área; classificar as plantas quanto ao habitat ocupado: se terrestre, de brejos e tipicamente aquáticas e suas prováveis origens geográficas.

\section{OBJETIVO ESPECÍFICO}

Realizar o levantamento florístico do complexo da Cascalheira.

\section{ÁREA DE ESTUDO}

O presente trabalho foi realizado em uma área fortemente modificada, situada à margem sul-mato-grossense do Rio Paraná nominada popularmente de Cascalheira. A área é banhada pelas águas do Reservatório de Jupiá - UHE "Eng. Souza Dias" Rio Paraná, município de Três Lagoas (20 47' 24.97"S; $51^{\circ} 42^{\prime} 20.0^{\prime \prime O}$ ), de 
propriedade da Companhia Energética de São Paulo (CESP), conforme Silva e Vilela, (2008). Localizada na intersecção dos rios Sucuriú e Paraná, originalmente coberta por vegetação típica de Cerrado (NETO, 2010), hoje o que se observa é um conjunto de lagoas de ressurgência entremeadas por vegetação secundária, como pode ser observado na Figuras 1, 2 e 3. A dimensão ocupada é de aproximadamente 6,60 ha.

Figura 1- Imagem de Satélite da Cascalheira

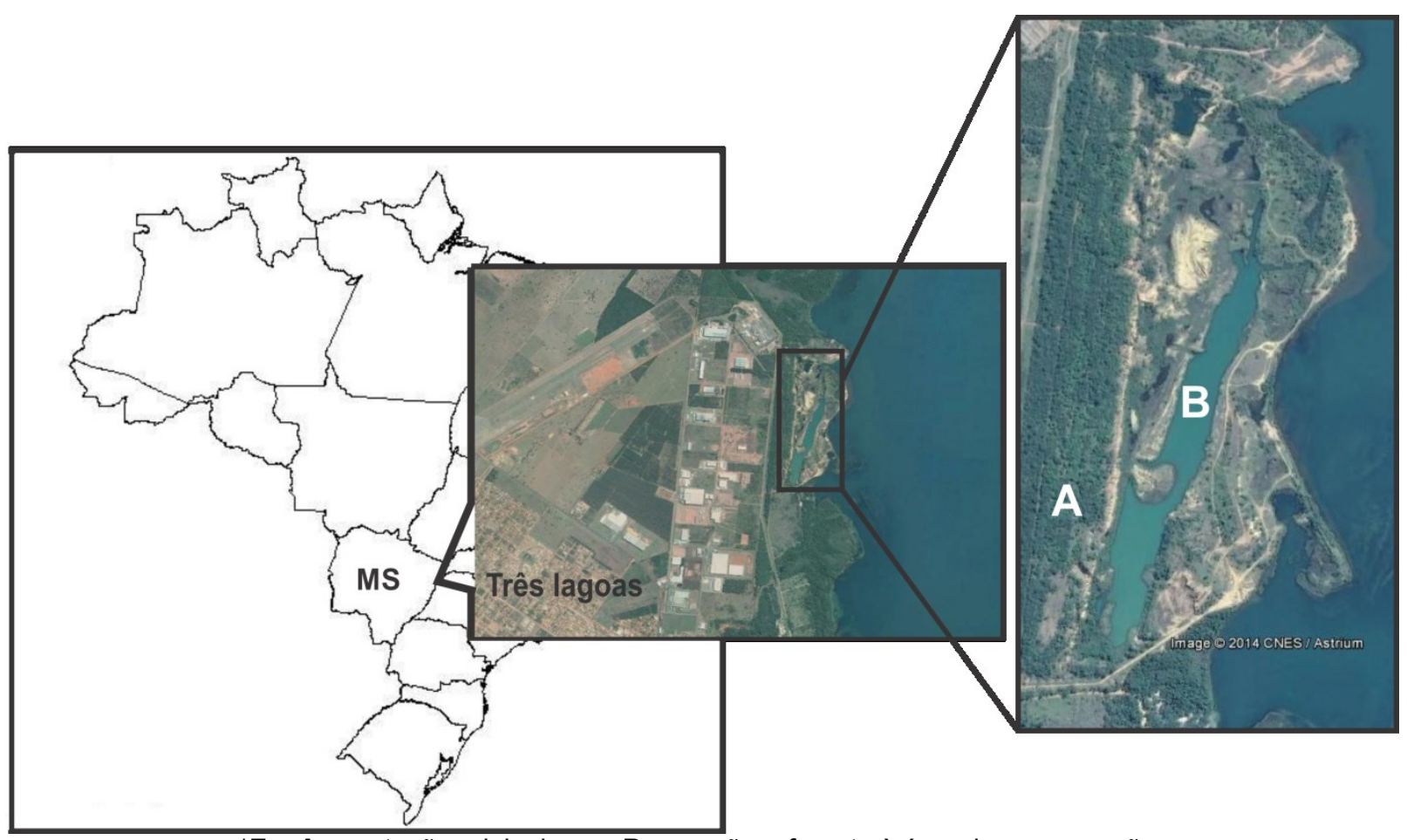

*Em A vegetação original e em $\mathbf{B}$ a porção referente à área de regeneração.

Fonte: Extraído e modificado dos sites Google ® Earth (@2014 Google - Imagens @2014 Digital Globe).

Figura 2- Vegetação original ao fundo

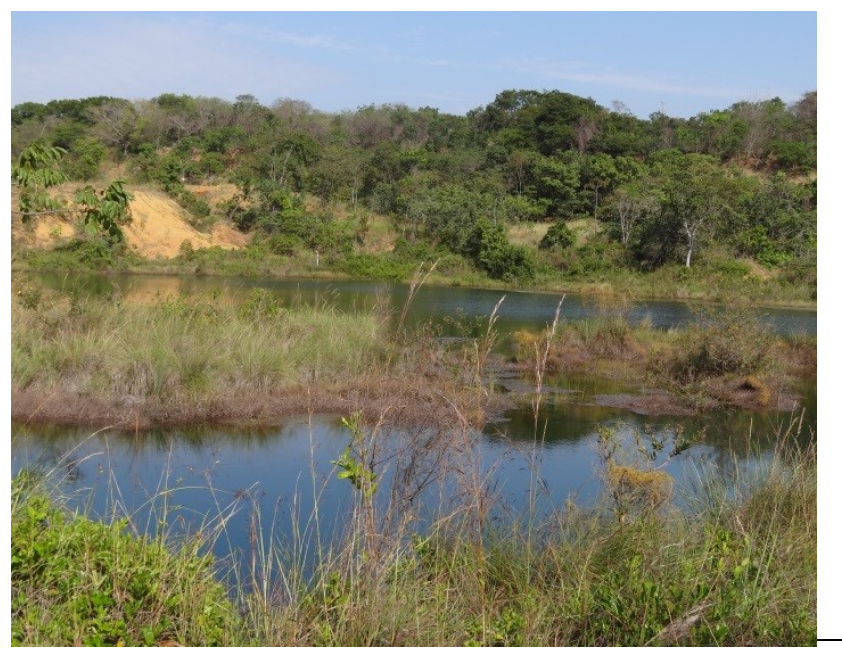

Figura 3- Lago com vegetação típica

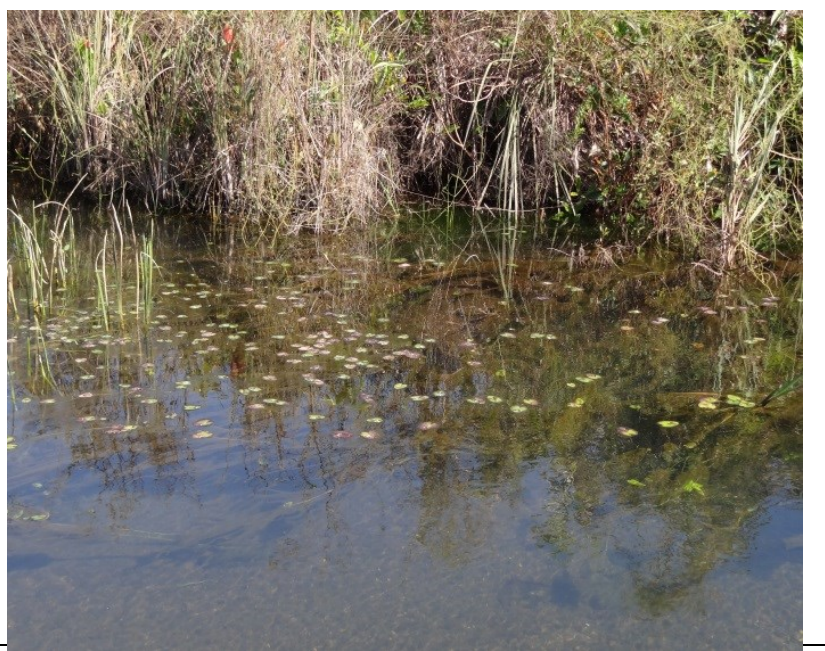




\section{Metodologia}

A classificação das espécies em nível de famílias botânicas seguiu o sistema APG III Angiosperm Phylogeny Group III (2009), que posiciona as famílias seguindo suas relações filogenéticas. As grafias dos nomes científicos e seus descritores seguiram os registros das bases de dados do Missouri Botanical Garden e The International Plant Names Index, ambos disponíveis no site The Plant List (2010). A identificação das Pteridophyta tiveram como base literária Souza et al. (1997) e Prado e Hirai (2012). Espécimes encontrados apenas em fases vegetativas aguardam identificação. As plantas, além da identificação botânica, foram agrupadas em terrestres, aquáticas e de brejos, bem como quanto suas origens ou ocorrências geográficas.

As coletas dos dados foram realizadas em campanhas trimestrais durante o ano de 2013 e a metodologia utilizada foi a de caminhamento, de acordo com Filgueiras et al. (1994), sempre levando em conta as proximidades com os corpos d'água. Os espécimes coletados foram herborizados de acordo com procedimento convencional e depositados no herbário CEUL da Universidade Federal do Mato Grosso do Sul. Espécies facilmente reconhecidas foram apenas fotografadas, alternativa sugerida por Judd et al. (2009).

\section{RESULTADOS E DISCUSSÕES}

Foram coletados 136 espécimes de plantas, dos quais 135 foram identificados até o nível de espécie e 01 até o nível de gênero. Os 136 espécimes distribuíram-se em 107 gêneros e em 50 famílias. As famílias mais numerosas foram Cyperaceae e Poaceae (Graminae) com 14 espécies cada; Fabaceae (Leguminosae) com 13 espécies; Melastomataceae com 09 espécies e Alismataceae com 05 espécies. Das famílias botânicas relacionadas, 28 apresentaram apenas uma espécie. As relações dos espécimes identificados são apresentadas na Tabela 1.

Tabela 1. Espécies identificadas em uma área denominada de Cascalheira, município de Três Lagoas-MS. 


\begin{tabular}{|c|c|c|c|}
\hline Família & Nome cientifico & Porte/hábito & $\begin{array}{l}\text { Ocorrência } \\
\text { Origem }^{11}\end{array}$ \\
\hline Alismataceae & Echinodorus grandiflorus (Cham. \& Schltdl.) Micheli & Aquática & BR \\
\hline Alismataceae & Echinodorus longipetalus Micheli & Aquática & BR \\
\hline Alismataceae & Limnocharis flava (L.) Buchenau & Aquática & BR \\
\hline Alismataceae & Sagittaria guayanensis Kunth & Aquática & AM \\
\hline Alismataceae & Sagittaria rhombifolia Cham. & Aquática & AMS \\
\hline Amaranthaceae & Alternanthera BRiana (L.) Kuntze & Terrestre & BR \\
\hline Anacardiaceae & Astronium fraxinifolium Schott & Terrestre & BR \\
\hline Annonaceae & Annona dioica A.St.-Hil. & Terrestre & BR \\
\hline Annonaceae & Xylopia aromatica (Lam.) Mart. & Terrestre & BR \\
\hline Annonaceae & Xylopia emarginata Mart. & Terrestre & BR \\
\hline Apocynaceae & Calotropis procera (Aiton) Dryand. & Terrestre & BR \\
\hline Apocynaceae & Mandevilla hirsuta (Rich.) K.Schum. & Terrestre & BR \\
\hline Araceae & Pistia stratiotes L. & Aquática & PAN \\
\hline Arecaceae & Acrocomia aculeata (Jacq.) Lodd. ex Mart. & Terrestre & $\mathrm{BR}$ \\
\hline Asteraceae & Bidens gardneri Baker & Terrestre & BR \\
\hline Asteraceae & Clibadium armanii (Balb.) Sch.Bip. ex O.E.Schulz & Terrestre & BR \\
\hline Asteraceae & Lessingianthus glabratus (Less.) H.Rob. & Terrestre & BR \\
\hline Asteraceae & Pterocaulon virgatum (L.) DC. & Terrestre & BR \\
\hline Asteraceae & Vernonanthura BRiana (L.) H.Rob. & Terrestre & BR \\
\hline Bignoniaceae & Cybistax antisyphilitica (Mart.) Mart. & Terrestre & BR \\
\hline Bignoniaceae & Fridericia florida (DC.) L.G.Lohmann & Terrestre & BR \\
\hline Bignoniaceae & Handroanthus chrysotrichus (Mart. ex DC.) Mattos & Terrestre & BR \\
\hline Bignoniaceae & Tabebuia insignis (Miq.) Sandwith & Brejo & BR \\
\hline Bignoniaceae & Pyrostegia venusta (Ker Gawl.) Miers & Terrestre & BR \\
\hline Cactaceae & $\begin{array}{l}\text { Praecereus euchlorus (F.A.C.Weber ex K.Schum.) } \\
\text { N.P.Taylor }\end{array}$ & Terrestre & BR \\
\hline Cabombaceae & Cabomba caroliniana A.Gray & Aquática & AM \\
\hline Combretaceae & Combretum duarteanum Cambess & Terrestre & BR \\
\hline Combretaceae & Terminalia glabrescens Mart. & Terrestre & BR \\
\hline Commelinaceae & Commelina diffusa Burm.f. & Terrestre & BR \\
\hline \multicolumn{4}{|c|}{ Continuação... Tabela 1} \\
\hline Família & Nome cientifico & Porte/hábito & $\begin{array}{l}\text { Ocorrência } \\
\text { Origem }^{11}\end{array}$ \\
\hline Commelinaceae & Commelina obliqua Vahl & Terrestre & $\mathrm{BR}$ \\
\hline Convolvulaceae & Ipomoea asarifolia (Desr.) Roem. \& Schult. & Terrestre & BR \\
\hline Convolvulaceae & Ipomoea cairica (L.) Sweet & Terrestre & BR \\
\hline Cyperaceae & Ascolepis BRiensis (Kunth) Benth. ex C.B.Clarke & Brejo & AMC/AMS \\
\hline Cyperaceae & Calyptrocarya glomerulata (Brongn.) Urb. & Brejo & AM \\
\hline Cyperaceae & Cyperus aggregatus (Willd.) Endl. & Brejo & AM \\
\hline Cyperaceae & Cyperus haspan L. & Brejo & AM \\
\hline Cyperaceae & Cyperus luzulae (L.) Retz. & Brejo & AM \\
\hline Cyperaceae & Cyperus odoratus L. & Brejo & AM \\
\hline Cyperaceae & Cyperus surinamensis Rottb. & Brejo & AMT \\
\hline Cyperaceae & Eleocharis capillacea Kunth & Brejo & AMS \\
\hline Cyperaceae & Eleocharis elegans (Kunth) Roem. \& Schult. & Brejo & AMC/AMS \\
\hline Cyperaceae & Fimbristylis complanata (Retz.) Link & Brejo & AMT/VMT \\
\hline Cyperaceae & Kyllinga brevifolia Rottb. & Brejo & PAN \\
\hline
\end{tabular}




\begin{tabular}{|c|c|c|c|}
\hline Cyperaceae & Kyllinga vaginata Lam. & Brejo & AMC/AMS \\
\hline Cyperaceae & Pycreus lanceolatus (Poir.) C.B.Clarke & Brejo & AF \\
\hline Cyperaceae & Scleria mitis P.J.Bergius & Brejo & AMC/AMS \\
\hline Dilleniaceae & Curatella americana L. & Terrestre & BR \\
\hline Dilleniaceae & Davilla elliptica A.St.-Hil. & Terrestre & BR \\
\hline Droseraceae & Drosera communis A.St.-Hil. & Brejo & BR \\
\hline Eriocaulaceae & Syngonanthus decorus Moldenke & Brejo & $\mathrm{BR}$ \\
\hline Euphorbiaceae & Cnidoscolus quercifolius Pohl & Terrestre & $\mathrm{BR}$ \\
\hline Fabaceae & Crotalaria lanceolata E.Mey. & Terrestre & $\mathrm{AF}$ \\
\hline Fabaceae & Dioclea violacea Benth. & Terrestre & $\mathrm{BR}$ \\
\hline Fabaceae & Erythrina crista-galli L. & Terrestre & BR \\
\hline Fabaceae & Indigofera hirsuta L. & Terrestre & BR \\
\hline Fabaceae & $\begin{array}{l}\text { Inga sessilis (Vell.) Mart. } \\
\end{array}$ & Terrestre & BR \\
\hline Fabaceae & Machaerium amplum Benth. & Terrestre & $\mathrm{BR}$ \\
\hline Fabaceae & Macroptilium lathyroides (L.) Urb. & Terrestre & AMT \\
\hline Fabaceae & Mimosa debilis Willd. & Terrestre & BR \\
\hline Fabaceae & Mimosa hirsutissima Mart & Terrestre & BR \\
\hline Fabaceae & Mimosa pellita Willd. & Terrestre & BR \\
\hline Fabaceae & Mimosa polycarpa Kunth & Terrestre & MAS/AMS \\
\hline Fabaceae & Mimosa somnians Willd. & Terrestre & BR \\
\hline Fabaceae & Sesbania virgata (Cav.) Pers. & Brejo & $\mathrm{BR}$ \\
\hline Gentianaceae & Chelonanthus alatus (Aubl.) Pulle & Brejo & BR \\
\hline Hydrocharitaceae & Egeria densa Planch. & Aquática & BR \\
\hline Lamiaceae & Hyptis mutabilis (Rich.) Briq. & Terrestre & BR \\
\hline Lauraceae & Cassytha filiformis $\mathrm{L}$. & Parasita & BR \\
\hline Lycopodiaceae & $\begin{array}{l}\text { Palhinhaea camporum (B. Øllg. \& P.G. Windisch) } \\
\text { Holub }\end{array}$ & Brejo & BR \\
\hline Lythraceae & Cuphea melvilla Lindl. & Brejo & BR \\
\hline Malpighiaceae & Byrsonima umbellata Mart. ex.A.Juss. & Brejo & BR \\
\hline Malvaceae & Ayenia tomentosa L. & Terrestre & BR \\
\hline Malvaceae & Guazuma ulmifolia Lam. & Terrestre & BR \\
\hline Malvaceae & Hibiscus sororius L. & Terrestre & BR \\
\hline \multicolumn{4}{|c|}{ Continuação... Tabela 1} \\
\hline Família & Nome cientifico & Porte/hábito & $\begin{array}{l}\text { Ocorrência } \\
\text { Origem }^{11}\end{array}$ \\
\hline Malvaceae & Luehea divaricata Mart. & Terrestre & BR \\
\hline Malvaceae & Luehea paniculata Mart. & Terrestre & BR \\
\hline Malvaceae & Melochia simplex A. St.-Hil. & Brejo & BR \\
\hline Malvaceae & Pavonia cancellata (L.) Cav. & Terrestre & BR \\
\hline Malvaceae & Pavonia guerkeana R.E. Fr & Terrestre & BR \\
\hline Malvaceae & Pseudobombax longiflorum (Mart. \& Zucc.) A.Robyns & Terrestre & BR \\
\hline Malvaceae & Waltheria indica $\mathrm{L}$. & Terrestre & AMT \\
\hline Marantaceae & Thalia geniculata L. & Brejo & AMT \\
\hline Mayacaceae & Mayaca madida (Vell.) Stellfeld & Aquática & AM \\
\hline Melastomataceae & Clidemia hirta (L.) D. Don & Brejo & BR \\
\hline Melastomataceae & Desmoscelis villosa (Aubl.) Naudin & Brejo & $\mathrm{BR}$ \\
\hline Melastomataceae & Macairea radula (Bonpl.) DC. & Brejo & BR \\
\hline Melastomataceae & Miconia chamissois Naudin & Brejo & BR \\
\hline Melastomataceae & Miconia theaezans Cogn. & Brejo & BR \\
\hline
\end{tabular}




\begin{tabular}{|c|c|c|c|}
\hline Melastomataceae & Microlicia isophylla DC. & Brejo & BR \\
\hline Melastomataceae & Rhynchanthera dichotoma (Desr.) DC. & Brejo & BR \\
\hline Melastomataceae & Tibouchina gracilis (Bonpl.) Cogn. & Brejo & BR \\
\hline Melastomataceae & Tibouchina stenocarpa (DC.) Cogn. & Brejo & $\mathrm{BR}$ \\
\hline Menyanthaceae & Nymphoides indica (L.) Kuntze & Aquática & $\mathrm{AM} / \mathrm{AF}$ \\
\hline Moraceae & Ficus insipida Willd. & Terrestre & $\mathrm{BR}$ \\
\hline Ochnaceae & Sauvagesia erecta L. & Brejo & BR \\
\hline Onagraceae & Ludwigia nervosa (Poir.) H.Hara & Brejo & BR \\
\hline Onagraceae & Ludwigia octovalvis (Jacq.) P.H.Raven & Brejo & BR \\
\hline Onagraceae & Ludwigia tomentosa (Cambess.) H. Hara & Brejo & BR \\
\hline Orchidaceae & Cyrtopodium paludicola Hoehne & Brejo & BR \\
\hline Phyllantaceae & Phyllanthus amarus Schumach. \& Thonn. & Brejo & BR \\
\hline Piperaceae & Piper aduncum L. & Terrestre & BR \\
\hline Piperaceae & Piper fuligineum Kunth & Terrestre & $\mathrm{BR}$ \\
\hline Poaceae & Andropogon bicornis L. & Terrestre & AM \\
\hline Poaceae & Andropogon leucostachyus Kunth & Terrestre & AMC/AMS \\
\hline Poaceae & Brachiaria decumbens Stapf & Terrestre & $\mathrm{AF}$ \\
\hline Poaceae & \begin{tabular}{|l|l} 
Digitaria inAMSaris (L.) Mez ex Ekman \\
\end{tabular} & Terrestre & AMS \\
\hline Poaceae & Echinochloa crus-galli (L.) P.Beauv. & Terrestre & PAN \\
\hline Poaceae & Eriochrysis cayennensis P.Beauv. & Brejo & AMC/AMS \\
\hline Poaceae & Hymenachne pernambucensis (Spreng.) Zuloaga & Terrestre & AMC/AMS \\
\hline Poaceae & Hyparrhenia rufa (Nees) Stapf & Terrestre & $\mathrm{AF}$ \\
\hline Poaceae & Melinis minutiflora P.Beauv. & Terrestre & $\mathrm{AF}$ \\
\hline Poaceae & Melinis repens (Willd.) Zizka & Terrestre & $\mathrm{AF}$ \\
\hline Poaceae & Panicum aquaticum Poir. & Brejo & AMC/AMS \\
\hline Poaceae & Paspalum notatum Flüggé & Brejo & AM \\
\hline Poaceae & Sacciolepis vilvoides (Trin.) Chase & Brejo & AMC/AMS \\
\hline Poaceae & Steinchisma decipiens (Nees ex Trin.) W.V.Br. & Brejo & AMS \\
\hline Pontederiaceae & Eichhornia azurea (Sw.) Kunth & Aquática & BR \\
\hline Pontederiaceae & Eichhornia crassipes (Mart.) Solms & Aquática & BR \\
\hline Pontederiaceae & Pontederia cordata L. & Aquática & AMS \\
\hline Pontederiaceae & Pontederia sagittata C.PresI & Aquática & AMC/BR \\
\hline \multicolumn{4}{|c|}{ Continuação... Tabela 1} \\
\hline Família & Nome cientifico & Porte/hábito & $\begin{array}{c}\text { Ocorrência } \\
\text { Origem }^{/ 1}\end{array}$ \\
\hline Primulaceae & Anagallis minima (L.) E.H.L.Krause & Brejo & BR \\
\hline Pteridaceae & Pityrogramma calomelanos (L.) Link & Brejo & BR \\
\hline Pteridaceae & Pityrogramma trifoliata (L.) R.M. Tryon & Brejo & BR \\
\hline Rubiaceae & Alibertia edulis (Rich.) A.Rich. ex DC. & Terrestre & BR \\
\hline Rubiaceae & Sipanea pratensis Aubl. & Terrestre & BR \\
\hline Rubiaceae & Spermacoce multiflora (DC.) Delprete & Terrestre & BR \\
\hline Salviniaceae & Salvinia auriculata Aubl. & Aquática & $\mathrm{BR}$ \\
\hline Salviniaceae & Salvinia biloba Raddi & Aquática & BR \\
\hline Sapindaceae & Serjania lethalis A. St.-Hil. & Terrestre & BR \\
\hline Solanaceae & Solanum paniculatum L. & Terrestre & BR \\
\hline Solanaceae & Solanum sp. & Terrestre & BR \\
\hline Thelypteridaceae & Cyclosorus interruptus (Willd.) H. Itô & Brejo & BR \\
\hline Typhaceae & Typha domingensis Pers. & Brejo & BR \\
\hline Urticaceae & Cecropia pachystachya Trécul & Terrestre/brejo & BR \\
\hline
\end{tabular}


Xyridaceae

Xyridaceae Xyris jupicai Rich. Xyris laxifolia Mart.

1- Ocorrência/origem, onde: $B R=$ Brasil, AMS = América Do Sul, AMT = América Tropical, PAN = Pantropical, AMC = América Central, $\mathrm{AM}=$ Américas, $\mathrm{AF}=$ África, $\mathrm{VMT}=\mathrm{VM}$ Temperado; símbolo "/ "= e: exemplo: AMC e AF

Ecologicamente, 68 espécies são terrestres (50,01\%), 52 de brejos $(38,23 \%)$ e $16(11,76 \%)$ espécies são tipicamente aquáticas. Uma única espécie Cassytha filiformis L. é semi-parasita. Quanto às origens geográficas, 99 (72,79\%) são de origem brasileira, 6 são africanas $(4,41 \%)$ e 31 são oriundas das Américas ( América do Sul, Central, Pantropical ) (22,79\%).

Observamos que na área em regeneração, $72,79 \%$ das espécies têm ocorrência ou origem pertencente ao Brasil, evidenciando que os espécimes encontrados no local em estudo pertencem à vegetação primária (Cerrado) ou ruderais, segundo Neto et al. (2010).

Dos 136 espécimes registrados, 14 destes pertencem à família Poaceae (Graminae) representando cerca de $10,29 \%$ do total das espécies identificadas. Mesmo sendo um valor relativamente pequeno em relação às outras espécies, a ocorrência das poáceas tornou-se importante para a regeneração natural, pois segundo Nóbrega et al. (2008), as gramíneas criam condições para o restabelecimento do solo, favorecendo a umidade para o desenvolvimento inicial, mesmo sujeitas às perturbações naturais e pressões antrópicas. De acordo com Chapman (1996), as gramíneas são apropriadas para a recomposição de áreas degradadas, atuando como pioneiras na sucessão ecológica. Se de um modo isto possibilita vantagem na ocupação de áreas em seu habitat original, por outro pode incrementar o grau de invasão de espécies alóctones. Dentre as espécies com essas características, pode-se destacar Melinis minutiflora (SOUZA et al., 2005); no entanto, na região em estudo problemas maiores poderão ser causados por espécies de Brachiaria, principalmente $B$. decumbens que ocorrem em praticamente qualquer área abandonada na referida região, conforme Neto (2010).

As espécies tipicamente aquáticas são, na maioria absoluta, originárias do Brasil ou do Continente Americano, o que representa e permite concluir que não há caráter invasor nos corpos d'água. 
A presença de algumas espécies arbóreas, como Cybistax antisyphilitica (Mart.) Mart.; Erythrina crista-galli L.; Handroanthus chrysotrichus (Mart. ex DC.) Mattos; Guazuma ulmifolia Lam.; Luehea divaricata Mart.; Astronium fraxinifolium Schott; Ficus insipida Willd. entre outras, pode ser remanescente de plantio, elaborado pela CESP nos finais do século passado, no entanto, como pode ser observado na Figura 4, o plantio inicial já mostra resultados de reprodução, principalmente para a espécie Luehea divaricata.

Figura 4 - Agrupamento de espécies arbóreas remanescentes de plantio pela CESP

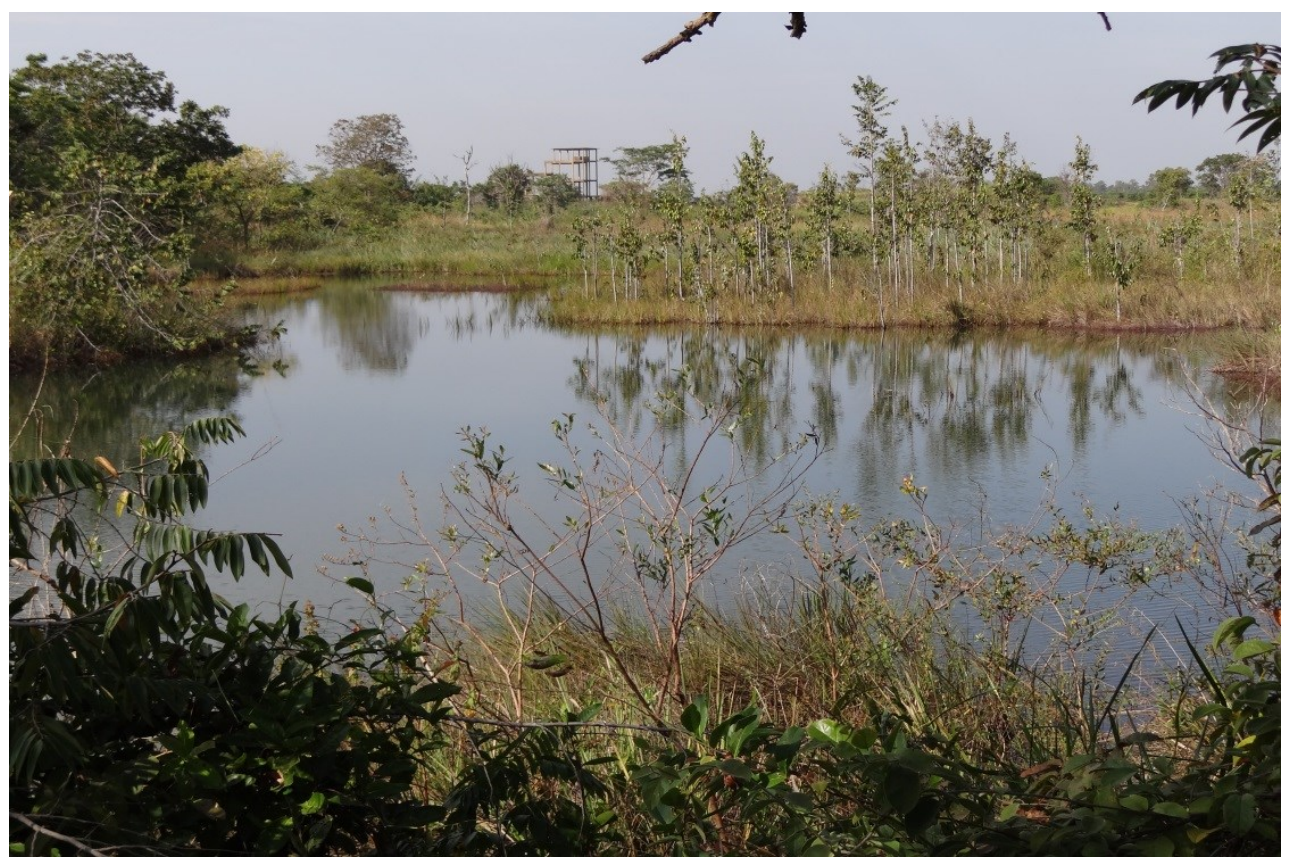

Fonte: (Neto, 2010)

\section{CONCLUSÃO}

Os resultados encontrados para o processo de regeneração natural demonstraram que a área em estudo apresenta condições para que as espécies nativas se estabeleçam, criando condições para o processo de sucessão ecológica. Analisar este tipo de processo em áreas de Cerrado é importante, pois possibilita compreender qual o grau de capacidade de regeneração natural em locais antropicamente modificados. Conservar às áreas com vegetação natural ou de grandes fragmentos próximos as áreas em processo de regeneração é de suma 
importância, uma vez que contribuem com estoques de propágulos o local poderá formar uma cobertura vegetal definitiva.

\section{REFERÊNCIAS}

ANGIOSPERM PHYLOGENY GROUP II. An update of the angyosperm phylogeny group classification for the orders and families of flowering plants: APG II. Botanical Journal of the Linnean Society, London, v.41, n.11, p.399-436, 2003.

APG III. 2009. An update of the Angiosperm Phylogeny Group classification for the orders and families of flowering plants: APG III. Botanical Journal of the Linnean Society16: 105-121.

CAMPELLO, M. C. et al, Seleção de Espécies para Recuperação de Áreas Degradadas por Meio da Formação de llhas de Vegetação, 1997.

CARVALHO, J. O. P. Structure and dinamic of a logged over Brazilian Amazoniam rain forest. 1992. 215p. Thesis (PhD Thesis). Univerty of Oxford, Oxford, 1982.

CHAPMAN, G.P. 1996. The biology of grasses. Wallingford, CAB International.

FARIA, H. H.; SÉRGIO, F. C.; GARRIDO, M. A. O. Recomposição da vegetação ciliar integrada à conservação de microbacia. Revista doInstituto Florestal, n.21, p.1-22, 2001.

FERREIRA, E. C. et al. Estabelecimento de mata ciliar às margens do reservatório da usina hidrelétrica de Camargos, MG. Ciência florestal, Santa Maria, v. 19, n. 1, p.69-81, 2009.

FERREIRA, W. C. et al. Regeneração natural como indicador de recuperação de área degradada a jusante da usina hidrelétrica de Camargos, MG. Revista Árvore [online]. 2010, v.34, n.4, p. 651660. ISSN 0100-6762.

FILGUEIRAS, T. S.; BROCHADO, A. L.; NOGUEIRA, P. E.; GUALA, G. F. Caminhamento: um método expedito para levantamentos florísticos qualitativos. Caderno de Geociências, Rio de Janeiro, v.12, n.4, p.39-43, 1994.

FONSECA, S. N.; RIBEIRO, J. H. C.; CARVALHO, F. A. Estrutura e diversidade da regeneração arbórea em uma floresta secundária urbana (Juiz de Fora, MG, Brasil). Floresta Ambient. 2013, v.20, n.3, p. 307-315. ISSN 2179-8087.

GOOGLE $^{\mathrm{TM}}$ Earth 2013. Google Inc @ $2013-$ Imagens $\odot 2014$ Digital Globe. [acesso em 03 jun. 2014]. Disponível em: http://earth.google.com/intl/pt/.

JUDD, W. S.; CAMPBELL, C. S.; KELLOGG, E. A.; STEVENS, P. F.; DONOGHUE, M. J. Sistemática vegetal: um enfoque filogenético. 3. ed. Porto Alegre: Artmed, 2009. $632 \mathrm{p}$.

NETO, M .J .Levantamento florístico e colonização micorrízica em dois remanescentes de cerrado típico e em plantas ruderais no município de Três Lagoas-MS / Maria José Neto. -- Ilha Solteira : [s.n.], 2010, 145 f. : il.

NETO, M. J. et al Plantas ruderais. Campo Grande, Ed. UFMS 2010, 358 p. ISBN 978-85-7613-2998 
NOBREGA, A. M. F; VALERI, S. V; PAULA, SILVA, R. C; SILVA, S. A. Regeneração natural em remanescentes florestais e áreas reflorestadas da várzea do rio Mogi-Guaçu, Luiz Antônio SP. Rev. Árvore [online]. 2008, vol.32, n.5, pp. 909-920. ISSN 0100.

PRADO, J.; HIRAI, R.Y. Diversidade de samambaias dos fragmentos florestais remanescentes da região noroeste do estado de São Paulo. In: Fauna e Flora de Fragmentos Remanescentes da Região Norte do Estado de São Paulo/editor, Orlando Necchi Júnior. Ribeirão Preto: Holos, Editora, 2012. $301 \mathrm{p}$.

RODRIGUES, R. R. Restauração de florestas tropicais: indicadores de avaliação e monitoramento vegetal. In: Simpósio Sobre Restauração Ecológica De Ecossistema Naturais, 1., 1999,Piracicaba. Anais...Piracicaba: Universidade de São Paulo/ESALQ/Departamento de Ciências Florestais, 1999. 8p.

SILVA, E. R. Caracterização Da Regeneração Natural Da Mata Ciliar Do Córrego Alegre, Município De Inhumas, Estado De Goías. 2005. 13 f. TCC (Graduação) - Curso de Engenharia Ambiental, Universidade Católica de Goiás, Goiânia, 2005.

SILVA, R. Z.; VILELA, M. J. A. Nidificação de Phrynops geoffroanus (SCHWEIGGER, 1812) (CHELONIA: CHELIDAE) na área do reservatório de Jupiá - Rio Paraná, Três Lagoas, MS. Estud. Biol., v.30, n.70;71;72, p. 107-115, 2008.

SILVEIRA, T. I.; MARANHO, L. T. Avaliação da regeneração natural da vegetação em área recuperada com biossólido. Revista Brasileira de Ciências Ambientais, São Paulo, v. 25, n. 1, p.6273, 2012.

SOUZA, A ; MORAES, M. G; RIBEIRO, R. C. L. F. Gramíneas do cerrado: carboidratos nãoestruturais e aspectos ecofisiológicos. Acta Bot. Bras. [online]. 2005, vol.19, n.1, pp. 81-90. ISSN 0102-3306.

SOUZA, M. L. L. Comunidade de Jupiá em Três Lagoas; MS: Estudo de caso com enfoque no turismo gastronômico como estratégia de desenvolvimento local. 2007. 142 f. Dissertação (Mestrado) - Curso de Pós-graduação em Desenvolvimento Local, Universidade Católica Dom Bosco, Campo Grande, 2007.

SOUZA, M.C.; CISLINSKI, J.; ROMAGNOLO, M.B. Levantamento florístico. In: Ana Emília Amato de Moraes Vazzoler; Angelo Antonio Agostinho; Norma Segatti Hahn. (Org.). A planície de inundação do alto rio Paraná. Maringá: EDUEM : Nupélia, 1997, v. , p. 343-368.

THE PLANT LIST (2010). Version 1. Published on the Internet; <http:;;www.theplantlist.org> (Acesso nos períodos de execução do trabalho). 